\title{
Asynchronous, parallel on-line classification of P300 and ERD for an efficient hybrid BCI
}

\author{
Hannes Riechmann and \\ Nils Hachmeister \\ CITEC \\ Bielefeld University, Germany \\ hriechma,nhachmei@techfak.uni-bielefeld.de
}

\author{
Helge Ritter \\ CITEC \\ CoR-Lab \\ Bielefeld University, Germany \\ helge@ techfak.uni-bielefeld.de
}

\author{
Andrea Finke \\ CoR-Lab \\ Bielefeld University, Germany \\ afinke@cor-lab.uni-bielefeld.de
}

\begin{abstract}
We propose an on-line hybrid BCI system that combines P300 and ERD. By employing both brain activity patterns (BAPs) in parallel and asynchronously, the system can issue different types of commands, for example, in robotic control scenarios. We present a method for reliably distinguishing between the two BAPs. We examined the level of false positives in $\mathrm{P300}$ classification while a subject tries to evoke an ERD. We found this level to be as low as for regular P300 trials. Our system thus assumes the presence of ERD whenever classification of all P300 symbols is negative. Empirical results indicate that subjects can achieve good control over the hybrid BCI. In particular, subjects can switch spontaneously and reliably between the two BAPs.
\end{abstract}

\section{INTRODUCTION}

Brain-computer interfaces (BCIs) are a rapidly evolving research field. This research focuses on restoring communication and manipulation capabilities in severely handicapped patients [1], [2]. One widely used technique for data acquisition in the field of BCIs is electroencephalography (EEG). It is affordable, safe and provides good temporal resolution. However, the signal-to-noise ratio is low and spatial resolution is limited compared to other techniques such as fMRI.

Different brain activity patterns (BAPs) have been identified in human EEG, some of them can be employed for BCIs. P300 [3], [4] and event-related desynchronization (ERD) [1] are two common examples of these BAPs. State-of-theart EEG-based BCIs mostly employ only one single BAP. Hybrid BCIs, for which this limitation is dropped, are a relatively new branch within the field of BCIs. According to [2], "a typical hybrid BCI is [...] composed of one BCI and another system (which might be another BCI)“. Hence, to construct a hybrid BCI one can utilize various data acquisition methods (e.g., EEG and fMRI). Alternatively, one can classify different BAPs (e.g. ERD and SSVEP) using only a single data acquisition method.

For example, in [5], an ERD-based BCI was combined with an SSVEP-based BCI to improve classification. Subjects had to concentrate on an SSVEP stimulus indicating left or right and, to evoke an ERD, had to perform motor imagery (MI) of the left or right hand accordingly and simultaneously. The mean classification accuracy of the hybrid approach was reported to be significantly better than for ERD and slightly better than for SSVEP alone. Furthermore, it was reported that a hybrid BCI could be a way to overcome BCI illiteracy. In [2], a NIRS- or ERD-based BCI was used as an on/off switch for an SSVEP-based BCI. The authors applied their hybrid BCI successfully for orthosis control and reported that the use of an on/off switch reduced false positives. In [6], a hybrid BCI combining an ERD-based BCI (using MI) with EMG measurements was evaluated.

All of these studies share the same central motivation: the additional information from one system is used to boost the classification accuracy of another. Although there are already useful applications for this, in our opinion the additional information can also be used to allow for more elaborate control.

Recently, [7] proposed a BCI combining P300 and ERD. The BCI system was used for a 2D cursor control task. One dimension was controlled by ERD and the other dimension was controlled using P300. In this way, reliably controlling arbitrary cursor movements was possible. In particular, the users did not find it difficult to use both BAPs in combination.

In the cursor-control system the two BAPs were used to increase the dimensionality of the system, but using P300 for the continuous control of the cursor position is not intuitive, quite tricky to handle and therefore rather slow. This approach neglects the discrete nature of P300-based BCI commands.

In our study, we use P300 and ERD within one hybrid BCI system. In contrast to previous studies, our system classifies the two BAPs completely asynchronously and exploits the advantages of the individual BAPs in order to support a wide range of possible applications and an intuitive control.

\section{ERPS AND ERDS IN A HYBRID SYSTEM}

A hybrid BCI system, by our definition, is a system that can classify multiple BAPs (e.g. P300, ERD, SSVEP, etc.) on-line and asynchronously. Asynchronous classification demands that data segments used for the classification of the different BAPs can overlap arbitrarily. This asynchronism, however, still allows for a joint use of the different classification results. This is called label fusion (see section IIIC). The asynchronous classification of different BAPs and optional fusion of the classification results presents one of the central aspects of our study. 
We demonstrate that the asynchronous, on-line classification for several BAPs is possible and, more importantly, that the user can yield competitive classification rates involving both BAPs and switching between them arbitrarily. Furthermore, we show that our system is capable to detect which of the BAPs the user intends to produce solely using the EEG data.

The control of a robotic device is a suitable scenario to demonstrate the benefits of a hybrid BCI system. For the control of robotic devices, decisions to be made via the BCI can be divided into two classes: (i) Select one out of a set of different options (e.g., different objects). These decisions will be referred to as discrete decisions. (ii) Controlling a continuous state of the robot (e.g., movement control). These decisions will be called continuous decisions subsequently. The choice of the BAPs used for this study is based on these two decision types: P300 is classically a way to choose between different options (discrete decisions) while ERD can intuitively be used to make continuous decisions, e.g., by considering the duration of the ERD.

\section{METHODS}

The methods used for classifying the BAPs are well established in the field of BCI and will thus be introduced only briefly. In addition, we have to address the question of deciding which BAP the user intends to produce.

\section{A. Computational Steps}

As a first step, bandpass filtering was applied. For P300 classification, data was filtered to a band from $0 \mathrm{~Hz}-10 \mathrm{~Hz}$. For ERD classification, the filter band was optimized for every subject individually on the training data.

PCA-feature extraction: Within the P300 classification system, a standard principal component analysis (PCA) [3], [8], [9] was applied to vectors containing the (linearized) temporal and spatial dimensions of the data. For our experiments, the number of output dimensions of the PCA was chosen to maintain at least $99.9 \%$ of variance of the subjects training data.

CSP-feature extraction: Within the ERD classification system, a common spatial pattern analysis (CSP) [1], [10] was applied for feature extraction. Subsequently, the bandpower for each channel was computed, according to [11].

FDA-classification: The classification was done by a standard linear classifier using the Fisher's discriminant analysis [12] to determine the weights.

\section{B. Parallel Classification Pipelines}

The point of interest of this study is the parallel and asynchronous classification of EEG data for different BAPs. The different BAPs require different methods and/or parameters to be used for preprocessing, feature extraction, and classification. Therefore, data has to be copied and then fed into different classification pipelines, applying the necessary computational steps. Fig. 1 shows the two classification pipelines used for ERD and P300 classification. The pipelines differ mainly in the feature extraction algorithms

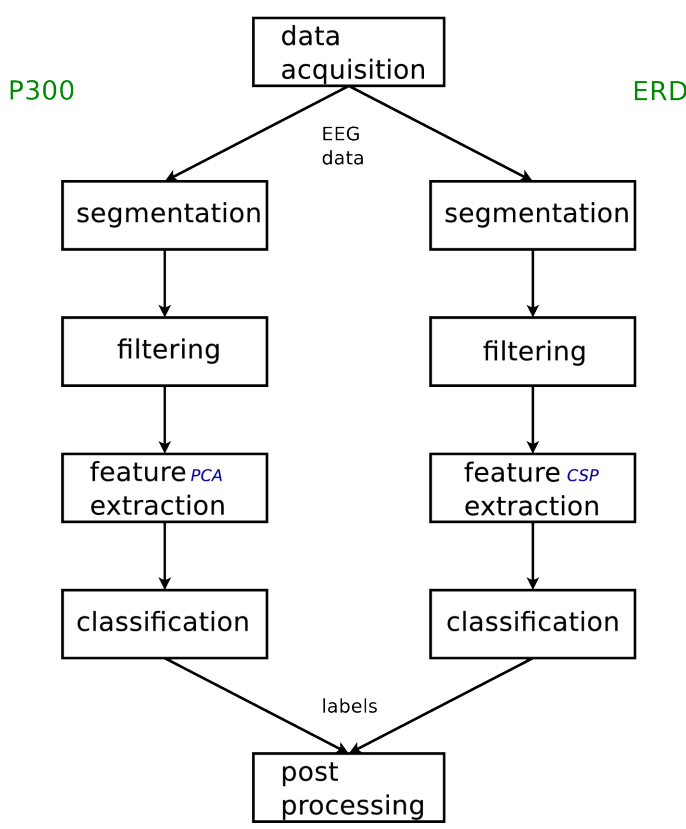

Fig. 1. Within the hybrid BCI system, EEG data is copied and fed into two different classification pipelines: one for P300 classification and one for ERD classification. Afterwards, the classification results of the individual pipelines are merged in a post-processing step (label fusion).

used. One of the results of the two pipelines is selected as total system result in the post-processing step.

\section{Label Fusion}

There are different ways to deal with the results of the individual pipelines. Our system is capable to (i) output any label of any pipeline as system output, (ii) wait until there is one label from each pipeline and then select one of the labels as system output. All other labels are discarded. This option will be called pattern selection. Although option (i) is rather simple, it is useful in many scenarios. However, option (ii) is the computationally more challenging one. In addition to classifying the individual BAPs, the system has to recognize which BAP the user intends to produce. Using such a system, any possible sequence of labels can be produced. Using our system, the user can either concentrate on any item of the P300 stimulus presentation or perform left or right hand MI. It is up to the system to determine which BAP the user actually employed and which target, associated with this BAP, corresponds to the users intention.

Our approach is guided by the following assumption: LDA output will be similar in two different cases: for an epoch associated with (i) a non-target flash and (ii) a flash for which the subject does not pay attention to the P300 stimulus presentation (e.g. because she/he is performing MI). In particular, if the subject does not pay attention to the P300 stimulus presentation at all, the LDA outputs will be negative for all epochs (except for classification errors).

Following this assumption, a mechanism was developed to decide whether P300 or ERD is the target BAP. If the score, i.e. the sum over the LDA outputs for one symbol, is negative for all P300 symbols, it is assumed that the target BAP is 
ERD. Thus, the ERD classification result is forwarded as the total system result. If the classification is positive for at least one of the P300 symbols, the symbol with the highest score is forwarded as the total system result.

\section{EXPERIMENTS AND RESULTS}

We conducted an experiment to evaluate our hybrid BCI system and the pattern selection approach.

\section{A. Experimental Setup}

The aim of the experiment was to evaluate the classification accuracy of our hybrid BCI system. A target, either one out of five P300 symbols (numbers from one to five) or one out of two motor imagery directions (left or right hand MI), was presented to the subject. Subjects were instructed to either concentrate on the P300 symbol or imagine hand movement accordingly. The hybrid BCI system computed one label in each of the two pipelines and subsequently selected one of these labels as total system result. This result was one of the seven potential targets for the subject and was presented to the subject to provide feedback. This procedure was repeated sixty times and system accuracy was computed.

Data was acquired using a gUSBamp (Guger Technologies) amplifier. 16 channels were equipped with $\mathrm{Ag} / \mathrm{AgCl}$ electrodes placed at Fz, Cz, Pz, Oz, F3, F4, C3, C4, C5, C6, CP3, CP4, P3, P4, PO7, PO8 according to the extended international 10-20 system. A ground and a common reference electrode were placed on the mastoids (A1 and A2). Electrode impedances were kept below $5 \mathrm{k} \Omega$. The amplifier sampled the EEG data at $256 \mathrm{~Hz}$, performed highpass filtering at $0.1 \mathrm{~Hz}$ and notch filtering at $48-52 \mathrm{~Hz}$. For the P300 stimulus presentation five symbols were used. One trial consisted of ten subtrials, in which each symbol flashed once in random order. The inter-stimulus interval (ISI) was set to $150 \mathrm{~ms}$. For each flash, a data segment of $800 \mathrm{~ms}$ starting at the beginning of the flash was generated. For the ERD pattern, a data segment of $4000 \mathrm{~ms}$ was generated, starting when the system indicated the start of a new trial. The first and the last $300 \mathrm{~ms}$ were discarded, in order to remove possible motor artifacts.

Four healthy subjects participated in the study. Each subject completed at least three MI training sessions of about thirty minutes on different days before the actual experiment. On the day of the experiment, a P300 training data set containing fifty trials was recorded. Subsequently, a shortened MI training was conducted until ERD classification was stable. Then, each subject performed one experimental session consisting of sixty trials. The target was presented for $10 \mathrm{~s}$ before the beginning of the trial alongside a countdown. During thirty trials the presented target was a P300 symbol and during thirty trials it was an MI direction. The targets were presented in random order. P300 stimuli were presented during any trial. The subjects were instructed not to pay attention to the P300 stimuli during MI trials.

\section{B. Experimental Results}

The results of the experiments are summarized in Table I. The upper third of the table shows the results obtained

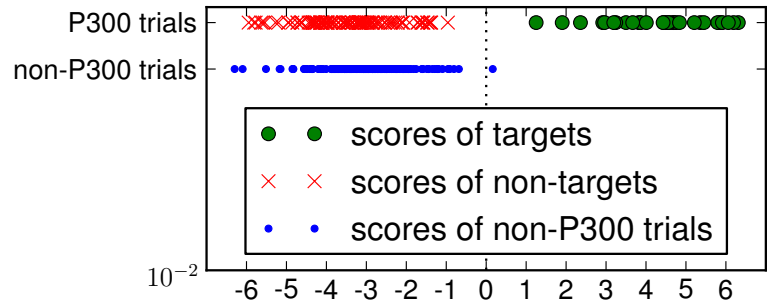

Fig. 2. P300 scores for subject S4 using ten subtrials. Non-target symbols and all symbols during non-P300 trials achieve similar scores. These are separable from scores for target symbols.

using ten subtrials for the P300 classification. The hybrid classification reaches an average classification accuracy of $82 \%$, every subject reached more than $70 \%$. For a correct classification the correct BAP must be selected and the classification pipeline for that BAP must classify the trial correctly. This means that errors in pattern selection and individual BAP classification accumulate. The average accuracy of the pattern selection was $95 \%$. The performance of the pattern selection strongly depends on the performance of the P300 classification.

Further analysis of P300 scores validates the basic assumption of the pattern selection approach. Fig. 2 shows all classification scores of the P300 classification for subject S4 using ten subtrials. The P300 scores during trials with an MI target (depicted as small dots) resemble the scores for nontarget symbols during P300 trials (depicted as crosses) and are separable from the scores for target symbols (depicted as large dots). These results apply for all subjects.

The classification of the ERD is more difficult. In general, we experienced that the MI performance of the individual subjects depends on their daily condition. Still, the hybrid system reached an average MI classification accuracy of $71 \%$. For comparison, the average classification accuracy during the MI training sessions is also shown. On average, ERD classification results during MI training and during the hybrid experiment differ by less than $1 \%$. This indicates that alternating between the two BAPs does not impede subjects' MI performance.

During the experiments ten subtrials were used for P300 classification. Later, we studied off-line the classification accuracy when using a dynamic subtrial limitation. All parameters for the dynamic subtrial limitation were computed subject-specifically using the P300 training data acquired before the experiment. The middle third of Table I shows that with an average of about five subtrials, the system looses only about $5 \%$ in accuracy. The correct BAP is computed in $90 \%$ of all trials. Fig. 3 depicts the relation of average number of subtrials and hybrid system accuracy in more detail. Three out of four subjects reach a satisfactory level of accuracy with an average of only four subtrials. At an average of seven subtrials, the accuracy is stable for all subjects. 
TABLE I

CLASSIFICATION RATES OF THE HYBRID BCI SYSTEM

\begin{tabular}{|c|c|c|c|c|c|c|}
\hline & Subject1 & Subject2 & Subject 3 & Subject 4 & Average & Remarks \\
\hline static P300 & & & & & & Ten subtrials for P300 and pattern sel. \\
\hline total hybrid system & 0.83 & 0.73 & 0.80 & 0.90 & $0.82 \pm 0.06$ & \\
\hline pattern selection & 0.95 & 0.93 & 0.95 & 0.98 & $0.95 \pm 0.02$ & Acc. in computing the correct BAP \\
\hline $\mathrm{P} 300$ & 1.00 & 1.00 & 1.00 & 1.00 & $1.00 \pm 0.00$ & Acc. of $\mathrm{P} 300$ pipeline, computed on $\mathrm{P} 300$ trials \\
\hline dynamic P300 & & & & & & Dynamic subtrial limitation \\
\hline total hybrid system & 0.80 & 0.70 & 0.75 & 0.84 & $0.77 \pm 0.05$ & \\
\hline pattern selection & 0.92 & 0.88 & 0.88 & 0.93 & 0.90 & Acc. in computing the correct BAP \\
\hline $\mathrm{P} 300$ & 0.97 & 0.97 & 1.00 & 0.97 & $0.98 \pm$ & Acc. of $\mathrm{P} 300$ pipeline, computed on $\mathrm{P} 300$ trials \\
\hline average no. subtrials & $6.43 \pm 1.22$ & $4.63 \pm 2.32$ & $5.05 \pm 2.13$ & $3.41 \pm 1.92$ & $4.88 \pm 1.08$ & Average number of subtrials used \\
\hline MI classification & & & & & & Independent from the number of P300 subtrials \\
\hline iment & 0.7 & 0.5 & 0.67 & 0. & 0.7 & Acc. of MI pipeline, computed on MI trials only \\
\hline MI training & 0.74 & 0.83 & 0.69 & 0.58 & $0.71 \pm 0.09$ & Acc. during pure MI training, for comparison \\
\hline
\end{tabular}

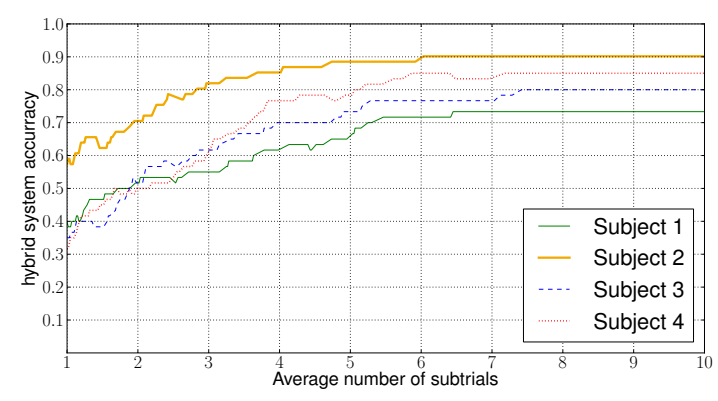

Fig. 3. Hybrid system accuracy in dependence of the average number of P300 subtrials when using dynamic subtrial limitation. The accuracy stabilizes at an average of seven subtrials.

\section{DISCUSSION AND CONCLUSION}

Our work shows that combining the P300 and the ERD patterns within a single BCI system is a promising approach for more flexibly controlling technical device, e.g. robots.

The accuracy of the hybrid system demonstrates that subjects were able to use and switch between the two BAPs as intended. We can therefore discard objections as being unsubstantiated that alternating between the non-volitional P300 and the volitional ERD patterns might have been cognitively too demanding. Indeed, average ERD classification rates hardly differ between the MI training and the more demanding hybrid experiment. In support of these results, all subjects reported that using the hybrid system was not more exhausting than using the ERD and P300 systems individually.

Our hybrid system is able to reliably distinguish between the two BAPs. Evaluation of the experimental data confirms the assumption that $\mathrm{P} 300$ scores are negative, when the subject performs MI. Accordingly, the correct pattern is chosen in almost every trial. In off-line analysis of the experimental data, we could show that the number of subtrials can be significantly reduced. Indeed, this number and, consequently, the duration of a trial could be halved, causing only a minor reduction in classification accuracy.

In conclusion, the performance of the hybrid system allows for the reliable control of devices such as robots. In the future, we plan to evaluate our system during control of a real robot.

\section{ACKNOWLEDGMENTS}

The study was partially funded by the German Research Council (DFG), EXC 277.

Andrea Finke gratefully acknowledges the financial support from Honda Research Institute Europe.

We thank Hendrik Koesling for his constructive comments.

\section{REFERENCES}

[1] J. Müller-Gerking, G. Pfurtscheller, and H. Flyvbjerg, "Designing optimal spatial filters for single-trial EEG classification in a movement task," Clinical neurophysiology, vol. 110, no. 5, pp. 787-798, 1999.

[2] G. Pfurtscheller, B. Z. Allison, G. Bauernfeind, C. Brunner, T. S. Escalante, R. Scherer, T. O. Zander, G. Müller-Putz, C. Neuper, and N. Birbaumer, "The hybrid bci," Frontiers in Neuroscience, vol. 4, 2010.

[3] F. Lotte and C. Guan, "An efficient p300-based brain-computer interface with minimal calibration time," 2009. [Online]. Available: HAL:http://hal.inria.fr/inria-00430563/en/

[4] C. J. Bell, P. Shenoy, R. Chalodhorn, and R. P. N. Rao, "Control of a humanoid robot by a noninvasive brain-computer interface in humans," Journal of Neural Engineering, vol. 5, no. 2, pp. 214-220, 2008.

[5] B. Z. Allison, C. Brunner, V. Kaiser, G. R. Müller-Putz, C. Neuper, and G. Pfurtscheller, "Toward a hybrid brain-computer interface based on imagined movement and visual attention," Journal of Neural Engineering, vol. 7, no. 2, 2010.

[6] R. Leeb, H. Sagha, R. Chavarriaga, and J. del R. Millán, "Multimodal Fusion of Muscle and Brain Signals for a Hybrid-BCI," in 32nd Annual International Conference of the IEEE Engineering in Medicine and Biology Society (EMBC'10), 2010.

[7] Y. Li, J. Long, T. Yu, Z. Yu, C. Wang, H. Zhang, and C. Guan, "An eegbased bci system for 2-d cursor control by combining mu/beta rhythm and p300 potential," Biomedical Engineering, IEEE Transactions on, vol. 57, no. 10, pp. 2495-2505, 2010.

[8] M. Thulasidas, C. Guan, and J. Wu, "Robust classification of eeg signal for brain-computer interface," Neural Systems and Rehabilitation Engineering, IEEE Transactions on, vol. 14, no. 1, pp. 24-29, 2006.

[9] S. Andrews, R. Palaniappan, A. Teoh, and L. chu Kiong, "Enhancing p300 component by spectral power ratio principal components for a single trial brain-computer interface," pp. 639-644, 2008.

[10] Y. Wang, S. Gao, and X. Gao, "Common spatial pattern method for channel selelction in motor imagery based brain-computer interface," in Engineering in Medicine and Biology Society, 2005. IEEE-EMBS 2005. 27th Annual International Conference of the, 2005, pp. 5392 $-5395$.

[11] B. Blankertz, G. Dornhege, M. Krauledat, K. Müller, and G. Curio, "The non-invasive Berlin Brain-Computer Interface: Fast acquisition of effective performance in untrained subjects," NeuroImage, vol. 37, no. 2, pp. 539-550, 2007.

[12] A. Finke, A. Lenhardt, and H. Ritter, "2009 special issue: The mindgame: A p300-based brain-computer interface game," Neural Netw., vol. 22, pp. 1329-1333, November 2009. 